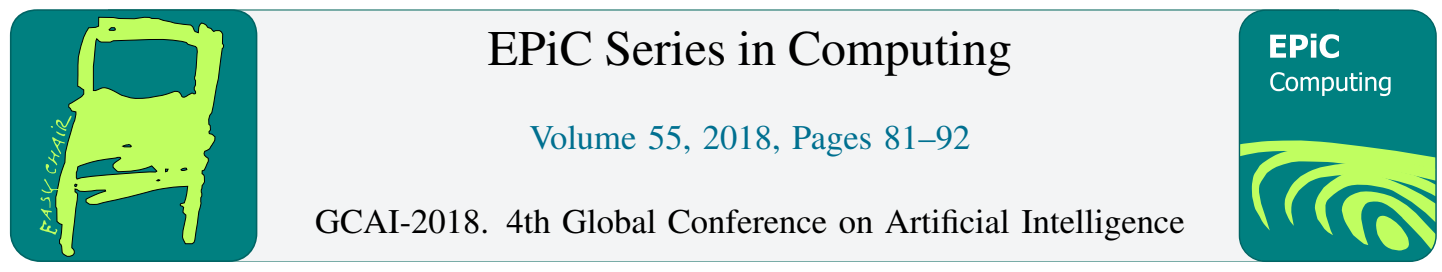

\title{
Genetic Algorithms for Scheduling and Optimization of Ore Train Networks
}

\author{
Ghulam Mubashar Hassan, Mark Reynolds, \\ Department of Computer Science and Software Engineering \\ The University of Western Australia \\ ghulam.hassan@uwa.edu.au,mark.reynolds@uwa.edu.au,
}

\begin{abstract}
Search and optimization problems are a major arena for the practical application of Artificial Intelligence. However, when supply chain optimization and scheduling is tackled, techniques based on linear or non-linear programming are often used in preference to Evolutionary Computation such as Genetic Algorithms (GAs). It is important to analyse whether GA are suitable for continuous real-world supply chain scheduling tasks which need regular updates.

We analysed a practical situation involving iron ore train networks which is indeed one of significant economic importance. In addition, iron ore train networks have some interesting and distinctive characteristics so analysing this situation is an important step toward understanding the performance of GA in real-world supply chain scheduling. We compared the performance of GA with Nonlinear programming heuristics and existing industry scheduling approaches. The main result is that our comparison of techniques here produce an example in which GAs perform well and is a cost effective approach.
\end{abstract}

\section{Introduction}

Supply chain management and associated optimization problems, involving both scheduling ahead and real-time decisions, are often both complicated and practically, financially and economically important: see, for example, case studies from the coal mining, airline crew, fleet vehicle routing and wine industries $[23,9,3]$.

Popular techniques for solving such complicated optimisation problems are those based on linear programming techniques and a hierarchical process in which multiple optimization problems are solved in a predefined sequence [4]. The Dantzig-Wolfe decomposition introduced in [12] uses delayed column generation to solve linear programming problems and is widely used to solve optimization problems [24]. Other variants of the decomposition technique are used to solve large optimization problems in industries such as airline crew scheduling [3], fleet vehicle routing [9], wireless mesh network resource allocation [5].

As optimization problems get larger or more complicated, these sorts of linear programming techniques (and variations with a mixture of some non-integers and/or some non-linearity) tend to need to involve heuristics, relaxation and branching and bounding [26] to find good results, albeit not necessarily global optima, within a reasonable amount of time. That raises a natural question about the search 
for optima in industrial applications. When, if at all, are the search problems complicated enough that traditional Artificial Intelligence (AI) search techniques such as, forms of evolutionary computation are better to use in practice than these operations research/mathematical optimization techniques?

In this paper, we look at a particular application domain, train scheduling on ore train networks, as an interesting and practical example arena for providing a local answer to this question about rival styles of optimization techniques.

There is already plenty of research on train scheduling problems. It is not possible to mention all of it: here are a few recent papers. Zhou and Zhong studied train scheduling for high speed passengers train [28]. Caprara et al proposed using a Lagrangian heuristic algorithm to schedule real-world trains [6]. Carey and Crawfords studied train scheduling at busy train stations [7]. Yuan and Hansen studied optimization of capacity of trains at train stations and their scheduling [27]. Abril et al proposed a distributed search method in the form of tree to solve a train scheduling problem [1]. Chung et al studied the Korean railway system and proposed a genetic algorithm based technique for train sequencing [10]. $\mathrm{Li}$ et al proposed a scheduling solution for trains depending on their global information [20]. Cheng and Yang studied the Taiwan railway system and proposed a Fuzzy Petri Nets approach for its control in abnormal or non-regular situations [8]. Lee and Chen proposed a heuristic approach for the train scheduling and its path optimization [19]. Fischetti et al studied Italian railways and optimizes train scheduling using non-linear and stochastic programming approaches [13]. Arenas et al proposed the use of genetic algorithm to solve Train Timetable Problem for annual railway operations service plan [2]. Gorman proposed a tabu search algorithm to find the weekly schedules for the freight railroad network [14] (tabu search is an iterative heuristic search technique which starts with a suitable solution and tries to improve it $[15,16])$. Gorman also compared the results with solution of genetic algorithms and found that tabu search works well for bigger sized problems.

These train scheduling studies are situated in large interconnected passenger and freight networks. Mining rail networks are different in important ways. For one, the networks are often much simpler, with one main route from each mine to the port. For example in the North-West of Western Australia there are four main private rail networks operating between company mines and their own ports: BHP Billiton, Rio Tinto, Fortescue Metals Group and Roy Hill. These are some of Australia's longest private rail networks.

To our knowledge there are few studies concentrating on solving the transportation scheduling problem via a rail network in the mining industry. Singh et al proposed a decision support system using mixed integer programming model [22]. The proposed system uses Hunter Valley Coal Australia as a case study and modeled its long term capacity expansion plan. The study focused on coal industry but did not propose any approach for optimizing train scheduling in coal transportation. Similarly, Kozan and Liu proposed a demand responsive decision support system for transportation of coal from order placement to delivery to the customer [17]. In this study all operations related to coal transportation including coal shipment, coal stockpiles and coal railway transportation are considered. The proposed decision support system is based on a non-linear programming approach and is applied on Queensland Australia's coal railway network. The study identified the railway network as a bottleneck to entire supply chain and evaluated the efficiency of the supply chain system with respect to the changes in number of trains used in the railway network. The study focused on time tabling the trains in order to optimize the entire supply chain involving train, stockpile and ship.

The other idiosyncratic characteristic of a mine rail network is that trains usually leave the mine when full, and leave the port when empty, rather than following a predetermined timetable [11]. A real-time train routing decision process is needed. To the best of our knowledge there is only one study which tackled the related issue of train scheduling depending on variable demand. Kuo et al studied the freight train scheduling having elastic demand [18]. A column generation based technique is proposed to optimize the freight train schedules to minimize the cost for carriers. In the study, a case study of pan- 
European network in analyzed. The study had a major constraint of considering the railway network to be unidirectional.

In this paper we consider two typical private mine train networks and attempt to optimize the throughput of the railways with the only control variables being the decisions to direct a train onto a siding to stop, and to stop (or not) trains approaching a junction. We conduct experiments with different numbers of trains available. There are many reasonable ways to formalize an objective function to represent throughput but in this preliminary study we just measure the time taken for all trains to leave the port empty and return full.

Our experiments compare optimization techniques based on the four main styles. We first use a standard industry approach that is implemented in a way to give priority to the trains going towards the port. In other words, loaded trains will get priority to have a non-stop journey which is our understanding of current industry practice. This approach is called Industry Approach. The second technique uses an approach where priority is given to the train which reaches the junction first. This approach is called Traditional Approach. The third technique is a standard Nonlinear Programming (NLP) approach where all possible decisions expected in future are considered among all trains over the railway network. The NLP approach is expected to always give the best results (global optimized solution) at the cost of computational complexity. The rival AI approach is just to use a very standard package genetic algorithm (GA) implementation to make the same control decisions. Because we work in real-time we curtail the searches to allow rescheduling every time an empty train leaves the port. After comparing all the four approaches, we simulated the network for 24 hours to analyze the long term improvement in the efficiency of scheduling the trains using different techniques.

The main contributions of this paper are as follows: a detailed case study of real-time optimization task for scheduling trains in real-life and important (but simple) train networks; a comparison of different approaches to this real-world problem; demonstration of significantly superior performance using the GA approach (in this case); and demonstrating the significant improvement in performance of the railway network by using the GA approach.

\subsection{Problem description}

In Western Australia, there are four major iron ore mining companies and each of them have their own private railway network for transportation of iron ore from their mines to Port Headlands. The collection of four private railway networks form Pilbara Railways. Each company has specific number of trains operating round the clock between their mines and Port Headlands. The number of trains may change to optimize the supply chain or maintenance.

Train scheduling is a critical problem in the supply chain of the iron ore mining industry. According to the norms of the industry, the trains are run close to maximum speed limit and loaded trains are mostly run nonstop from mine to port. The railway network is mostly single track with multiple sidings for crossing of trains. The decision of crossing of the trains has the simple criteria that unloaded trains are stopped on sidings to ensure the nonstop journey of loaded trains from mine to port [21]. This may decrease the overall cost and time of the train transportation operation. There can be multiple reasons for industry to run loaded trains nonstop from mine to port and important reasons are mentioned below which are expected to add extra cost and time in the operations.

- It makes the crossing decisions simple and avoids accidents or blockage of railway track.

- The loaded trains take longer time to accelerate to maximum speed limit as compared to the unloaded trains.

- The siding tracks may deteriorate quickly if loaded trains are stopped on them. 


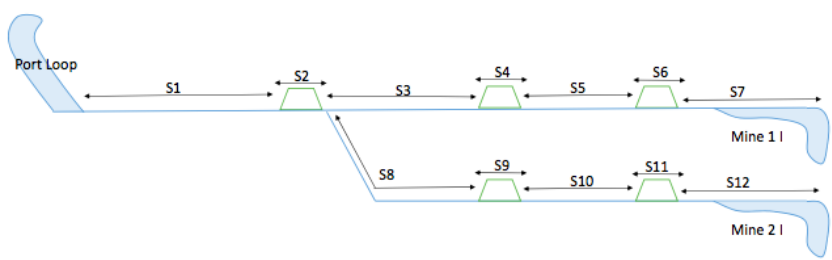

Figure 1: Sample iron ore railway network schematic.

In this study we propose that both loaded or unloaded trains may be stopped in order to optimize the overall time and cost of the train transportation operation. From the research of the area of Western Australia, it is found that all iron ore mines have higher altitude than the port and loaded trains are usually running down the hill. Therefore, the loaded train, which is heavy, will accelerate quickly downhill due to the gravity while unloaded trains will be taking longer time when accelerating up the hill. This is further discussed in the case study in next section of the article.

A sample iron ore railway network is shown in Figure 1. It contains railway tracks for two mines linked to a single port. There are two sections which are common between the two different tracks where one section has sidings for crossing of trains. There are five sections independent to each track which include two sidings on each track. Each train departing the port needs to know its destination mine. Each siding must have the length greater than the length of the train and must have one or more than one extra track to let a train stop on it. All sections of the railway network are represented by unique numbers such that Section 1 is represented by 'S1', Section 2 is represented by 'S2', and so on. Trains do not stop at the mines or port but slow down for loading and unloading respectively. Each train has either the status of 'loaded' or 'unloaded'. Each train takes some time to cross a point which depends on the length and speed of the train. Each train starts its journey from port as 'unloaded' train and ends its journey at port as 'loaded' train. The train knows its destination mine at the time of starting its journey and its status is 'unloaded' which changes to 'loaded' at the mine.

\section{Proposed Model}

The time taken by a train to complete its round trip is given by $T_{j}$ which is equal to the sum of the times taken by the train to cross each section on the railway network between port and destined mine. Each train will cross each section twice: once loaded and once unloaded. Subscript $j$ represents the train number.

$$
T_{j}=\sum_{i=1}^{m}\left(T_{s l_{i}}+T_{s u_{i}}\right)
$$

where $T_{s l}$ and $T_{s u}$ represent the time taken by the loaded and unloaded train respectively to cover a section and $i$ and $m$ represent the section number and total number of sections respectively.

In this study, we are trying to minimize the total time $T$ taken by all the trains to complete their journeys and reach back to the port loaded. It is represented as:

$$
T=\sum_{i=1}^{n} T_{i}
$$

where subscript $i$ represents the number of trains running on the railway network. 
Four different techniques are used to calculate $T$ as mentioned earlier. All techniques are used to simulate the network in advance by generating decisions of crossings of trains and minimize Equ. 2. The techniques are implemented in Java and the Jenetics library [25] is used for genetic algorithms.

In our model we propose to calculate the total time $T$ whenever a train starts its journey. Train demand is variable in the iron ore mining industry and are dispatched as per demand and availability. Using Traditional and Industry approaches, the decisions are easy to be implemented in real time while using NLP and genetic algorithms, the decisions are needed to be simulated in advance in quick manner to ensure that all decisions for the network are available before the time of the decision. The NLP approach simulates all possible decisions to find global minima while genetic algorithms are run for small time and it may happen that global minima can not be found. However, as the railway networks for iron ore mining industries are not very complicated and small, it is expected that a reasonable optimized solution will be obtained using standard GA in short time.

Following are the rules which are followed for experiments

- Each train leaving the port must know its destination.

- The journey for each train will start from a point on Section 1 which is linking to the port with status 'unloaded'.

- The journey for each train will stop with status 'loaded' at the same point on Section 1 from where it started its journey

- The status of the train changes from 'unloaded' to 'loaded' at the mine during its round trip journey.

- Each train needs to complete a round trip from port to mine and back to port.

- Each siding on the railway network can accommodate single or multiple trains depending on the length of the siding and length of the train.

- The length of the train is taken as $3 \mathrm{~km}$ for the sample iron ore railway network and length of the siding is taken as $6 \mathrm{~km}$. Each single track section is $60 \mathrm{~km}$ long.

- If two trains are found to be on a section in opposite direction then this will lead to crash of trains and system will stop all trains (simulations).

- The maximum speed limit for a train on the railway network is taken as $60 \mathrm{~km} / \mathrm{h}$.

- Each train traveling on the railway network is accumulating its $T_{j}$ which will keep on accumulating till its journey finishes.

- The total time $T$ is calculated for all the trains traveling on the network which have started the journey. Any train on the port will not be considered till it starts its journey.

- Each time when a train starts its journey, $T$ is calculated for the network. Therefore, one train on the railways network (one which is starting its journey) will have its $T_{j}=0$ while all other trains will have their own respective $T_{j}$.

For the NLP approach, all possible decisions of trains crossings needs to be simulated. It is observed that each train will cross another train once over its journey and each crossing will involve two decisions where either of the trains can be stopped. This shows that the total number of decisions $D$ will be dependent on the number of trains as: 


$$
D=2^{k} \quad \text { and } \quad k=\sum_{i=1}^{n-1} i
$$

where $n$ represents the total number of trains on the railway network. Thus NLP approach simulate the network with $D$ number of scenarios to find the global minima of $T$.

For the GA approach, the decisions to stop the trains is dependent on the genetic algorithms. Different possible decisions of train crossings are taken over entire railway network till all trains complete their journey and total time $T$ is calculated. The genetic algorithm simulate different decision scenarios to minimize Equ. 2. A chromosome genotype in this study consists of a sequence of boolean decisions where 0 and 1 represent the stopping of unloaded and loaded train respectively. The length of chromosome is $k$ and maximum number of possible decision is $D$ for $n$ trains as mentioned in Equ. 3. The altering of the offspring population is performed by mutation probability taken as 0.3 and crossover probability as 0.6 . Initial population is taken as 100 while the termination criteria for the evolution is 100 generations. The objective function is to minimize $T$ as presented in Equ. 2 and the minimum $T$ is selected as the optimal total time taken by all the trains present on the railway network to complete their journey.

For the evaluation of each approach, different numbers of trains are used in the railway networks and total time $T$ is found. For each selected number $n$ of trains, we conducted 100 simulations and calculated the average total time $T$. In each simulation, random positions of trains on the railway network are generated in such a manner that one train is about to start its journey from the port while rest of the trains are present on the railway network. The time taken by the randomly placed trains on the network is considered randomly in a range where minimum time taken is considered to be the time taken by the train traveling nonstop and is calculated by dividing the distance of the train which it is expected to cover by the maximum speed of the train and maximum time is $20 \%$ of the minimum time added to the minimum time.

After proving the concept of using GA as preferred technique, we conducted another set of experiments where we ran trains nonstop for 24 hours. The trains returning the port start their journey back to mine with their status changed from 'unloaded' to 'loaded'. All approaches are tested except the NLP approach due to its increasing computation time with the increase in number of decisions.

\subsection{Case study: Roy Hill}

Roy Hill's railway network is taken as a case study for our study because the details are available online [21]. The iron ore railway network schematic of Roy Hill is presented in Figure 2. The railway network is $344 \mathrm{~km}$ single lane with four sidings for crossing of trains. Each siding is approximately $3.2 \mathrm{~km}$ long and is sufficient for a train to stop. The heavy haul railway is built to support the transportation of 55Mtpa of iron ore. Roy Hill is using 4 trains transporting iron ore to port nonstop from mine. Each train is estimated to have payload of more than 31000 tonnes of iron ore. This shows that any improvement (decrease) in total time $T$ will significantly increase the revenue for Roy Hill.

As discussed earlier, it is not always optimal to stop an unloaded train to make the journey of loaded train nonstop. It can be observed from the information presented by Roy Hill that the payload of iron ore on each train will generate the acceleration due to gravity around $4500 \mathrm{~km} / \mathrm{hr} r^{2}$ on the ramp having an angle of $10^{\circ}$ due to its weight. This shows that loaded train will quickly accelerate to reach its maximum allowed speed and efficiency of overall system will not be significantly degraded if loaded trains are stopped to optimize the train scheduling. 


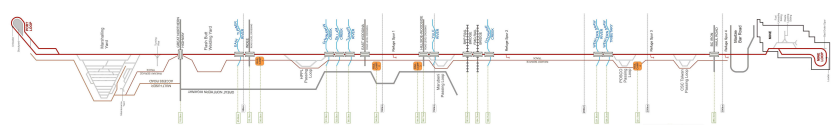

Figure 2: Roy Hill iron ore railway network schematic [21].

Table 1: Average Total time $T$ for sample railway network.

\begin{tabular}{|c|c|c|c|c|}
\hline \multicolumn{6}{|c|}{ Average total time $T$ in minutes } \\
\hline Trains & Industry & Traditional & NLP & GA \\
\hline 3 & 1650.17 & 1636.88 & $\mathbf{1 6 0 2 . 4 3}$ & $\mathbf{1 6 0 2 . 4 3}$ \\
4 & 2256.32 & 2195.57 & $\mathbf{2 1 2 8 . 8 9}$ & $\mathbf{2 1 2 8 . 8 9}$ \\
5 & 2863.01 & 2790.03 & $\mathbf{2 6 7 4 . 0 4}$ & $\mathbf{2 6 7 4 . 0 4}$ \\
6 & 3473.87 & 3371.19 & $\mathbf{3 2 1 1 . 9 8}$ & 3212.01 \\
7 & 4092.86 & 3957.96 & $\mathbf{3 7 5 3 . 6 5}$ & 3754.37 \\
8 & 4758.25 & 4571.24 & N/A* & $\mathbf{4 2 9 7 . 8 3}$ \\
\hline
\end{tabular}

* NLP had $2^{28}$ scenarios and was computational expensive

\section{Results and discussion}

The results for running the simulations using all considered techniques are calculated. The average total time $T$ for the sample railway network using all considered techniques is presented in Table 1 . The average round trip for a train on the sample railway network is plotted in Figure 3. It is found that Traditional Approach provided better results as compared to Industry Approach. In addition, Genetic Algorithms (GA) and NLP approaches provider better results as compared to Traditional and Industry approaches. This is due to the fact that NLP and GA approaches are able to foresee the impact of current decisions on future decisions. Comparing NLP and GA approaches, it is observed that GA either provides the same results or very close to NLP Approach. The NLP Approach simulating 8 trains on the sample network had $2^{28}$ different scenarios to simulate which was found to be computationally very expensive and experiment was not conducted for this scenario. However, it is expected in the light of earlier results that NLP approach would give the global minima on the cost computational complexity and GA approach would provide the results close to NLP approach.

It is expected that raising the number of trains increases the complexity of the train scheduling and crossing. The results show that the NLP Approach performs slightly better compared to GA Approach especially when number of trains are higher than 5 . However, the computation time is significantly increased due to the increase in the number of scenarios as mentioned in Equ. 3.

The average round trip time as mentioned in Figure 3 for a train increases with the increase in number of trains and is highest when eight trains are running on the sample network. The increase in the slope of average time for a train with the increase in number of trains using GA and NLP approaches is very low as compared to the increase in average time taken by a train using Traditional or Industry approaches. The average round trip time for a train is approximately equal for GA and NLP approaches.

Similarly, for the case study of Roy Hill railway network, the average total time $T$ using all analyzed approaches are presented in Table 2. The average round trip for a train on Roy Hill's railway network are plotted in Figure 4. The trend of the results is found to be similar as observed for the case of sample railway network. It is observed that the average round trip time for a train on Roy Hill network increases sharply with the increase in the number of trains as compared to the case of sample railway network. It is because Roy Hill's railway network has longer sections, a single mine and four sidings only. The increase in number of trains to five means that each single track has a train traveling on it. The results 
Average time taken by a train over sample network for single loop

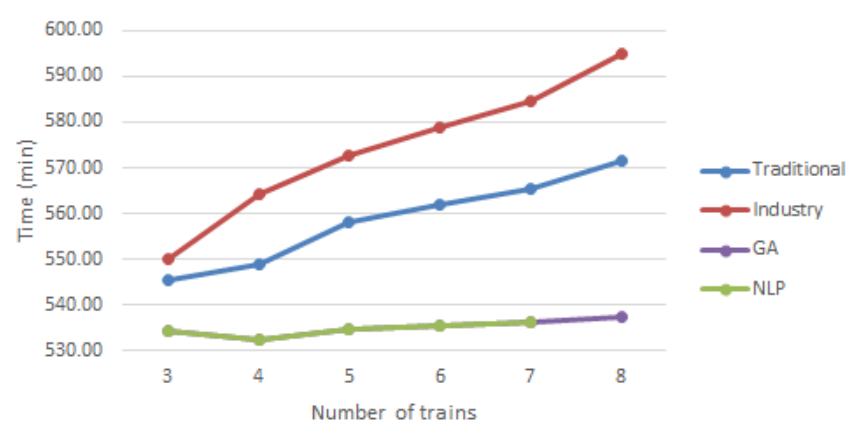

Figure 3: Average time taken by a train for a round trip journey on sample railway network. (Both GA and NLP approaches took approximately same time and are overlapping)

Table 2: Total time $T$ for Roy Hill's railway network.

\begin{tabular}{|c|c|c|c|c|}
\hline \multicolumn{6}{|c|}{ Average total time $T$ in minutes } \\
\hline Trains & Industry & Traditional & NLP & GA \\
\hline 3 & 2297.41 & 2216.01 & $\mathbf{2 0 8 0 . 6 1}$ & $\mathbf{2 0 8 0 . 6 1}$ \\
4 & 3201.11 & 3062.97 & $\mathbf{2 7 7 4 . 5 2}$ & $\mathbf{2 7 7 4 . 5 2}$ \\
5 & 4071.88 & 3882.28 & $\mathbf{3 4 7 6 . 8 8}$ & 3476.90 \\
6 & 5031.21 & 4780.72 & $\mathbf{4 1 6 0 . 4 9}$ & 4189.72 \\
7 & 5907.00 & 5587.73 & $\mathbf{4 8 7 0 . 7 6}$ & 4959.08 \\
8 & 6838.49 & 6448.34 & N/A* & $\mathbf{5 7 7 9 . 8 4}$ \\
\hline
\end{tabular}

* NLP had $2^{28}$ scenarios and was computational expensive

for 8 trains using NLP Approach are not available because it takes too long to generate $2^{28}$ different scenarios. The results of GA and NLP approaches are close to each other or the same and perform better than Traditional and Industry approaches.

Average time taken by a train time over Roy Hill's network for single loop

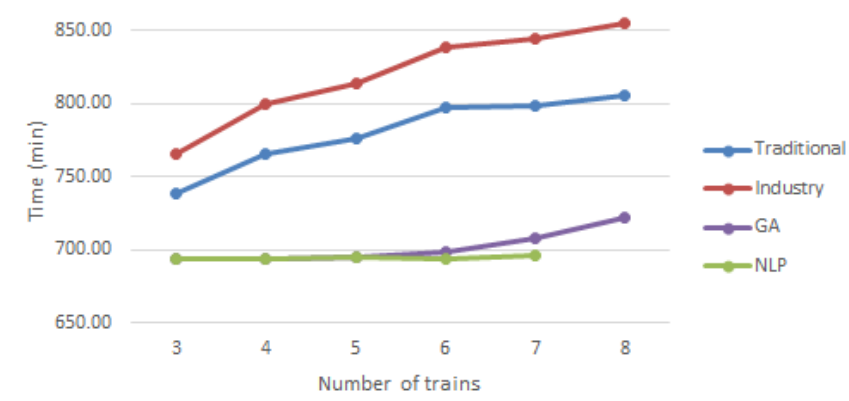

Figure 4: Time taken by a train for a round trip journey on Roy Hill's railway network. (Both GA and NLP approaches took approximately same time and overlapping in some part of the graph) 
Table 3: Average total distance covered by trains on Sample railway network.

\begin{tabular}{|c|c|c|c|}
\hline \multicolumn{4}{|c|}{ Average total covered distance in Kms } \\
\hline Trains & Industry & Traditional & Genetic Algorithm \\
\hline 3 & 4303.69 & 4870.46 & $\mathbf{4 9 5 2 . 2 0}$ \\
4 & 5423.53 & 6394.95 & $\mathbf{6 5 7 3 . 2 3}$ \\
5 & 6519.83 & 7898.56 & $\mathbf{8 1 8 3 . 2 0}$ \\
6 & 7551.51 & 9246.26 & $\mathbf{9 6 6 0 . 6 4}$ \\
7 & 8763.26 & 10725.49 & $\mathbf{1 1 3 5 9 . 2 8}$ \\
8 & 9776.38 & 11971.67 & $\mathbf{1 2 8 1 7 . 3 8}$ \\
\hline
\end{tabular}

Average distance covered by a train on sample network in 24 hours

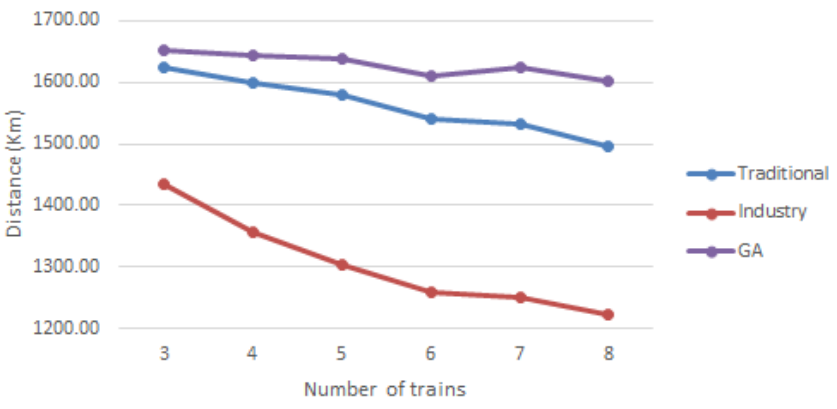

Figure 5: Average distance covered by a train in 24 hours on Sample railway network.

\subsection{Time based continuous experiment}

The results of the above experiments proved our proposed concept of using GA approach in scheduling trains for iron ore railway networks which are small, single path and have few crossings. In the next phase, we conducted experiments to run the system continuously for 24 hours where 'loaded' trains returning to the port start their journey towards the mine again after unloading itself. The status of the train changes to 'unloaded' again after reaching the port and scheduling system is simulated again for all decisions because a new 'unloaded' train is departing from the port. All approaches are simulated except NLP Approach because number of scenarios increased significantly due to the increase in the number of decisions involved over 24 hours of tested time. 100 experiments are simulated for different number of trains for both Sample and Roy Hill's railway network.

The average total distance covered by all trains over sample network in 24 hours for 100 simulated experiments is presented in Table 3. The graph for average distances covered by a train in 24 hours over sample railway network using different approaches is shown in Figure 5. As expected it is observed that average total distance covered by all trains increases with the increase in the number of trains running over the Sample railway network. However the average distance covered by a train over Sample railway network decreases with the increase of the number of train on the railway network. It can be observed from Table 3 and Figure 5 that the GA approach outperforms both Traditional and Industry approaches. The results also show that running long time simulations of 24 hours makes evident that the performance of Industry Approach is significantly lower than other approaches.

The experiments were also conducted on Roy Hill's railway network and average distances covered by all trains in 24 hours are presented in Table 4 . The average distance covered by a train over Roy 
Table 4: Average total distance covered by trains on Roy Hill's railway network.

\begin{tabular}{|c|c|c|c|}
\hline \multicolumn{4}{|c|}{ Average total time $T$ in minutes } \\
\hline Trains & Industry & Traditional & Genetic Algorithm \\
\hline 3 & 4897.94 & 5704.94 & $\mathbf{5 8 9 3 . 3 7}$ \\
4 & 6216.25 & 7365.20 & $\mathbf{7 5 2 9 . 8 3}$ \\
5 & 7627.49 & 8702.30 & $\mathbf{9 2 1 8 . 0 0}$ \\
6 & 8780.90 & 10318.42 & $\mathbf{1 0 7 7 8 . 7 9}$ \\
7 & 9964.88 & 11134.48 & $\mathbf{1 1 9 5 6 . 4 3}$ \\
8 & 11444.60 & 12709.71 & $\mathbf{1 3 3 4 3 . 5 7}$ \\
\hline
\end{tabular}

Average distance covered by a train on Roy Hill's network in 24 hours

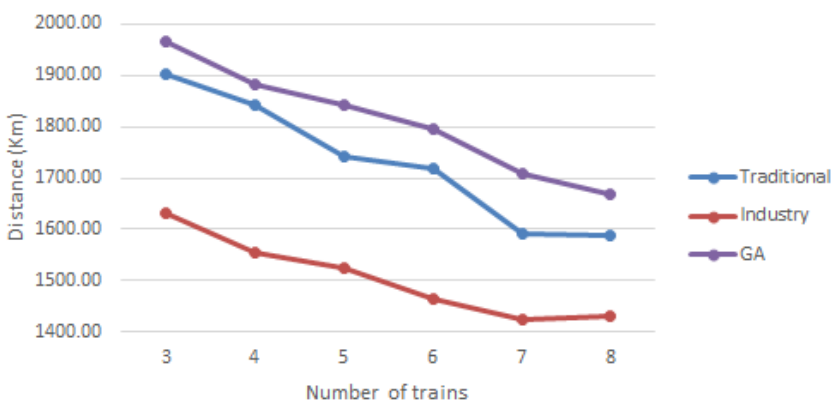

Figure 6: Average distance covered a train in 24 hours on Roy Hill's railway network.

Hill's railway network in 24 hours are presented in Figure 6. The results for Roy Hill's railway network were found to be similar to the case of Sample railway network. The performance of GA Approach is found to be better than other approaches.

\section{Conclusion}

We have presented a case study of a real-world real-time optimization task in supply chain management.

The case study involves the special situation of a private iron ore train network. These are quite simple networks with essentially one main route from each mine (of a few different ones) down to a single port. However, there are long sections of single track so that passing in different directions is done via sidings. We sought to optimize the throughput of the network with a fixed number of trains available only making decisions about the routing, halting and re-starting of trains in and out of sidings and into junctions. Because there is no schedule of train departures from the mines or the port, there is an important real-time element to this problem. Of course, the problem is one of serious financial importance to the mining company and the local economy.

The purpose of the experiments was to compare the performance of typical non-linear programming, operations research style approach to these problems with the less frequently used evolutionary computation styles. We just used a simple iterative version of each to run the real-time control. We used a few typical networks, one an actual network and we varied the number $n$ of trains available across the experiments. We compared the times to achieve a round trip of all $n$ trains from port back to port.

The result was a demonstration of superior performance using the GA approach. In addition, results 
show that iron ore industry should improve their scheduling system for railway network because all analyzed approaches performed better than the Industry Approach.

In future work we intend to extend these experiments to include ships' scheduling on the port and run simulations over multiple days to have a more realistic model.

\section{References}

[1] Montserrat Abril, Miguel A Salido, and Federico Barber. Distributed search in railway scheduling problems. Engineering Applications of Artificial Intelligence, 21(5):744-755, 2008.

[2] Diego Arenas, Rémy Chevrier, Said Hanafi, and Joaquin Rodriguez. Solving the Train Timetabling Problem, a mathematical model and a genetic algorithm solution approach. In 6th International Conference on Railway Operations Modelling and Analysis (RailTokyo2015), Tokyo, Japan, March 2015.

[3] Ralf Borndörfer, Uwe Schelten, Thomas Schlechte, and Steffen Weider. A column generation approach to airline crew scheduling. In Operations Research Proceedings 2005, pages 343-348. Springer, 2006.

[4] P. Brandimarte and M. Calderini. A hierarchical bicriterion approach to integrated process plan selection and job shop scheduling. International Journal of Production Research, 33(1):161-181, 1995.

[5] Antonio Capone, Giuliana Carello, Ilario Filippini, Stefano Gualandi, and Federico Malucelli. Solving a resource allocation problem in wireless mesh networks: A comparison between a cp-based and a classical column generation. Networks, 55(3):221-233, 2010.

[6] Alberto Caprara, Michele Monaci, Paolo Toth, and Pier Luigi Guida. A lagrangian heuristic algorithm for a real-world train timetabling problem. Discrete applied mathematics, 154(5):738-753, 2006.

[7] Malachy Carey and Ivan Crawford. Scheduling trains on a network of busy complex stations. Transportation Research Part B: Methodological, 41(2):159-178, 2007.

[8] Yung-Hsiang Cheng and Li-An Yang. A fuzzy petri nets approach for railway traffic control in case of abnormality: Evidence from taiwan railway system. Expert Systems with Applications, 36(4):8040-8048, 2009.

[9] Eunjeong Choi and Dong-Wan Tcha. A column generation approach to the heterogeneous fleet vehicle routing problem. Computers \& Operations Research, 34(7):2080 - 2095, 2007.

[10] Ji-Won Chung, Seog-Moon Oh, and In-Chan Choi. A hybrid genetic algorithm for train sequencing in the korean railway. Omega, 37(3):555-565, 2009.

[11] Jean-Francois Cordeau, Paolo Toth, and Daniele Vigo. A survey of optimization models for train routing and scheduling. Transportation science, 32(4):380-404, 1998.

[12] George B. Dantzig and Philip Wolfe. Decomposition principle for linear programs. Operations Research, 8(1):101-111, 1960.

[13] Matteo Fischetti, Domenico Salvagnin, and Arrigo Zanette. Fast approaches to improve the robustness of a railway timetable. Transportation Science, 43(3):321-335, 2009.

[14] Michael Francis Gorman. An application of genetic and tabu searches to the freight railroad operating plan problem. Annals of Operations Research, 78(0):51-69, 1998.

[15] Fred Glover. Tabu search-part i. ORSA Journal on computing, 1(3):190-206, 1989.

[16] Fred Glover. Tabu search part ii. ORSA Journal on computing, 2(1):4-32, 1990.

[17] Erhan Kozan and Shi Qiang Liu. A demand-responsive decision support system for coal transportation. Decision Support Systems, 54(1):665-680, 2012.

[18] April Kuo, Elise Miller-Hooks, and Hani S Mahmassani. Freight train scheduling with elastic demand. Transportation Research Part E: Logistics and Transportation Review, 46(6):1057-1070, 2010.

[19] Yusin Lee and Chuen-Yih Chen. A heuristic for the train pathing and timetabling problem. Transportation Research Part B: Methodological, 43(8):837-851, 2009.

[20] Feng Li, Ziyou Gao, Keping Li, and Lixing Yang. Efficient scheduling of railway traffic based on global information of train. Transportation Research Part B: Methodological, 42(10):1008-1030, 2008. 
[21] RoyHill. Roy hill — rail, 2017. [Online; accessed 18-January-2017].

[22] Gaurav Singh, David Sier, Andreas T. Ernst, Olena Gavriliouk, Rob Oyston, Tracey Giles, and Palitha Welgama. A mixed integer programming model for long term capacity expansion planning: A case study from the hunter valley coal chain. European Journal of Operational Research, 220(1):210 - 224, 2012.

[23] Anu Thomas, Jayendran Venkateswaran, Gaurav Singh, and Mohan Krishnamoorthy. A resource constrained scheduling problem with multiple independent producers and a single linking constraint: A coal supply chain example. European Journal of Operational Research, 236(3):946 - 956, 2014. Vehicle Routing and Distribution Logistics.

[24] François Vanderbeck and Laurence A. Wolsey. Reformulation and Decomposition of Integer Programs, pages 431-502. Springer Berlin Heidelberg, Berlin, Heidelberg, 2010.

[25] Franz Wilhelmstutter. Jenetics: Java genetic algorithm library, 2016. [Online; accessed 18-January-2017].

[26] Laurence A Wolsey. Integer programming john wiley \& sons. New York, NY, 4, 1998.

[27] Jianxin Yuan and Ingo A Hansen. Optimizing capacity utilization of stations by estimating knock-on train delays. Transportation Research Part B: Methodological, 41(2):202-217, 2007.

[28] Xuesong Zhou and Ming Zhong. Bicriteria train scheduling for high-speed passenger railroad planning applications. European Journal of Operational Research, 167(3):752 - 771, 2005. Multicriteria Scheduling. 